\title{
Use of LOINC and SNOMED CT with FHIR for Microbiology Data
}

\author{
Eugenia RINALDI ${ }^{\mathrm{a}, 1}$, Julian $\mathrm{SAAS}^{\mathrm{b}}$ and Sylvia THUN ${ }^{\mathrm{b}}$ \\ ${ }^{a}$ Charité Universitätsmedizin \\ ${ }^{\mathrm{b}}$ Berlin Institute of Health
}

\begin{abstract}
Infectious diseases due to microbial resistance pose a worldwide threat that calls for data sharing and the rapid reuse of medical data from health care to research. The integration of pathogen-related data from different hospitals can yield intelligent infection control systems that detect potentially dangerous germs as early as possible. Within the use case Infection Control of the German HiGHmed Project, eight university hospitals have agreed to share their data to enable analysis of various data sources. Data sharing among different hospitals requires interoperability standards that define the structure and the terminology of the information to be exchanged. This article presents the work performed at the University Hospital Charité and Berlin Institute of Health towards a standard model to exchange microbiology data. Fast Healthcare Interoperability Resources (FHIR) is a standard for fast information exchange that allows to model healthcare information, based on information packets called resources, which can be customized into so-called profiles to match use case- specific needs. We show how we created the specific profiles for microbiology data. The model was implemented using FHIR for the structure definition, and the international standards SNOMED $\mathrm{CT}$ and LOINC for the terminology services.
\end{abstract}

Keywords. FHIR, terminology, standard, LOINC, SNOMED CT, Infection control, Pathogen infectious diseases, microorganism, HiGHmed

\section{Introduction}

\subsection{Background}

Antimicrobial resistance is one of the major emerging infectious disease-related threats worldwide. There is a strong need to increase the scientific knowledge, coordinate efforts and promote data sharing to support secondary use of medical data. [1]

Hospital data are often collected in disparate proprietary systems with little standardization, which impedes data exchange and data integration. [2] To develop infrastructure for the integration of clinical data from patient care and medical research in Germany, the German Ministry of Education and Research launched the Medical Informatics Initiative (MII). [3] The MII, through the Medical Informatics consortium HiGHmed, addresses this goal also in the field of Infection Control. In HiGHmed, eight university hospitals have agreed to share their infection-related data to enable analyses

${ }^{1}$ Eugenia Rinaldi, Charité University Hospital, Charitéplatz 1, 10117 Berlin, Germany; E-mail: eugenia.rinaldi@charite.de. 
from different sources with the aim to detect potentially dangerous germs as early as possible and identify their transmission routes within and between hospitals.

Within the MII, in order to achieve syntactic and semantic interoperability, the adoption of international healthcare standards was recommended. Charité University hospital and Berlin Institute of Health have been particularly involved in the use of interoperability standards for the HiGHmed Use Case Infection Control. In this article, we will describe the work done for the modeling the microbiology data of the use case Infection Control adopting the standards FHIR (Fast Healthcare Interoperability Resources) with the terminology systems SNOMED CT (Systematized Nomenclature of Medicine Clinical Terms) and LOINC (Logical Observation Identifiers, Names, and Codes).

\subsection{Requirements}

In cooperation with domain experts, HiGHmed partners have created a minimum dataset of information to be shared within the use case Infection Control. For interoperability purposes, this data needs to be modeled according to the standard FHIR and the pertinent terminology bindings need to be implemented.

It is therefore necessary to create an information model that could be used within the entire HiGHmed community for the microbiology dataset using the FHIR standard. Moreover, wherever appropriate, the FHIR elements representing the microbiology data should be bound to SNOMED CT and LOINC terminologies.

\section{State of the art}

MII-participating university hospitals and their partners have formed consortia. These are tasked with developing strategies for shared data use and exchange. At present, meaningful data sharing and data analysis is slowed down by the use of disparate datasets and proprietary nonstandard systems in the different hospitals.

HiGHmed university hospitals seek to harmonize data to enable cross-institutional access, analytics and sharing of information. Their aim is to improve patient care and enhance clinical research in the field of Infection Control. MII consortia are working on different use cases in Germany but all with the aim to better exploit digitalization of medicine to improve healthcare and research. All consortia will make use of the FHIR standard in combination with standard terminologies to facilitate shared data use and exchange. The general acceleration in the deployment of electronic health record systems and the growing need for digitalization in healthcare has facilitated the use of terminologies and their wide adoption in the clinical community. Two of them, SNOMED CT and LOINC, have become international standards and seem the most appropriate to describe laboratory tests and results. [4,5]

\section{Concept}

The international standards organization Health Level Seven (HL7) created FHIR as the standard for healthcare data exchange, and it was identified by the MII as the standard to be used for modeling the data within the initiative. 
FHIR organizes information into packets, called resources, that can be retrieved individually while maintaining relationships to each other. Profiles are developed from these resources to customize them to specific use cases. In FHIR profiles, restrictions can be enforced on resources, data types can be specified, and terminology bindings can be established.[6]

The overall concept of the work presented here consists in designing FHIR profiles that represent the information contained in the HiGHmed microbiology dataset and making them available on a public platform such as Simplifier. Simplifier is a FHIR registry where FHIR projects can be published, viewed, and downloaded. The available MII general profiles can also be found on Simplifier. [7] For example, a general Laboratory Module with an Observation profile was made public by MII but it could not be used for Microbiology as it only allowed for quantitative results. We therefore submitted a request to the MII development community to include the quantitative data type for the Observation.value.

The FHIR resources that we used to profile the microbiology data were: Observation, Diagnostic Report, ServiceRequest and Specimen. The Observation resource is used to represent all laboratory examination data and its results. Specimen includes all the details relevant to the sample analyzed. ServiceRequest includes information on the order for laboratory examinations. DiagnosticReport summarizes all results together. The microbiology dataset of the HiGHmed use case includes primarily the examinations required to investigate the organism found in the sample. For this reason, we will mainly focus on the Observation and Specimen profiles. However, what is presented here is not the only possible FHIR representation of the microbiology information but a model proposal. For example, the choice of using the FHIR element hasMember seemed the most appropriate to group organism information together. The other FHIR grouping possibilities include the use of the component element which should be employed when there is only one method, one observation, one performer, one device, and one time and therefore seemed too restrictive for our use case. The element derivedFrom is meant to be used when one result derives from another result and in our case, a more general connection between the results seemed more fitting. The validity of the presented model can be confirmed only once a general Microbiology Module by the MII will be available.

\section{Implementation}

The relation between the structure and the semantics of data in FHIR is built-in within the resources and therefore needs to be addressed when modeling the information profiles.

Consequently, one important part of profiling the FHIR resources is to link its elements to the most fitting terminology systems and value sets. LOINC provides universal identifiers for laboratory tests, and SNOMED CT provides universal identifiers for organisms, substances, diseases, and other findings that may be recorded in the medical record or identified in test results. [8]

The microbiology dataset includes type of sample material and the site of collection. Sample material can be coded with SNOMED CT's Specimen hierarchy. The specimen collection site would typically come from SNOMED CT Body structure codes. However, some of the data that was provided by the HiGHmed partner sites, could not be covered with Specimen codes completely. In particular, there are no specimen codes for anaerobic or aerobic blood cultures. SNOMED CT does have these codes in the Procedures 
hierarchy though (e.g. $409612006 \mid$ Anaerobic blood culture (procedure) $\mid$ and 409611004 $\mid$ Aerobic blood culture (procedure)|). SNOMED CT separates the type of specimen e.g. $119297000 \mid$ Blood specimen (specimen) $\mid$ and the method that is used in a laboratory to analyze it e.g. 409611004 |Aerobic blood culture (procedure)|. This is furthermore aligned with how information is modeled in FHIR Observation resources. There can be references to Specimen resources that may use SNOMED codes and there is a method element that can bind to SNOMED procedure codes. The result of the test is reported through the value element. An example is shown in figure 1.

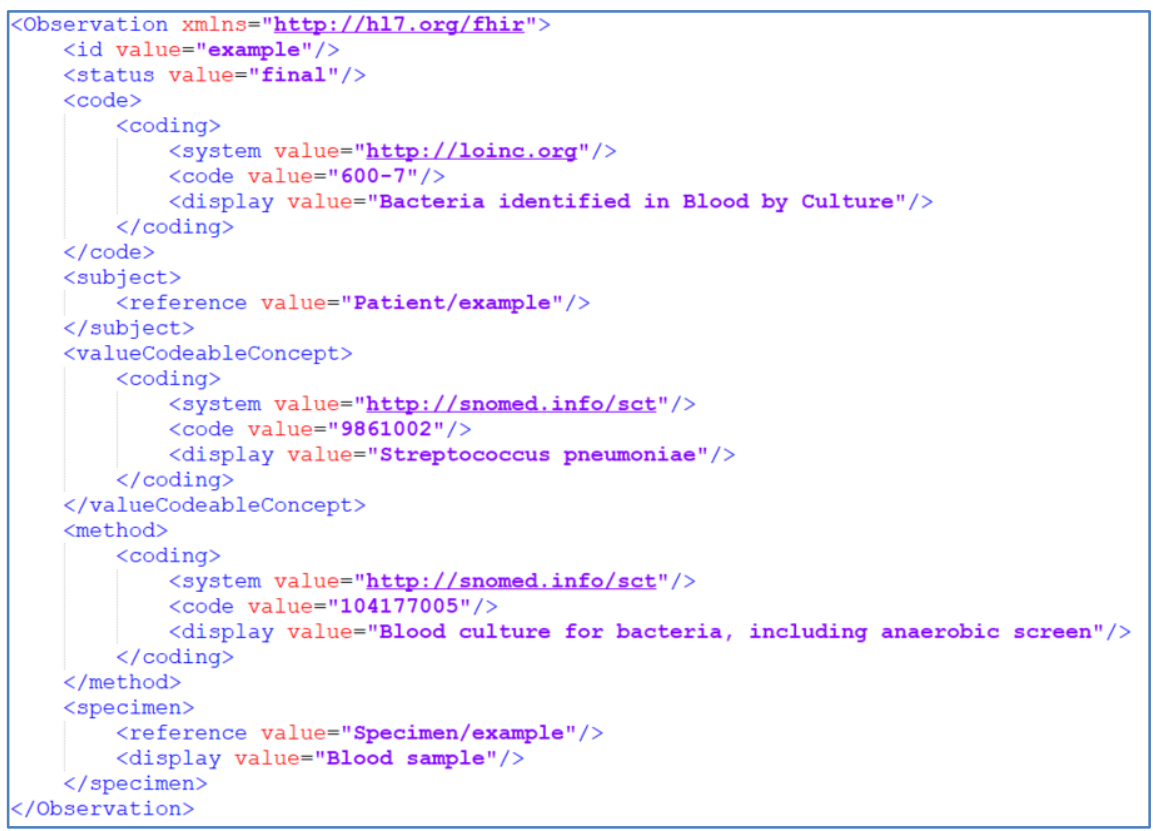

Figure 1: Example FHIR Observation resource for microbiology

The code element binds typically to a LOINC code that describes the performed test. Looking at the relevant LOINC test codes for microbiology, we can find two main categories

- Codes for tests identifying the presence of an organism (e.g. LOINC 43409-2 Bacteria identified in Isolate by Culture)

- Codes for tests measuring the quantity of an organism presence (e.g. LOINC 20774-6 Colony count [Units/volume] in Unspecified specimen by Visual count)

The first category expects a non-numerical result; it could be the name of an organism or a LOINC ordinal type of answer such as detected/not detected. LOINC defines as "ordinal" observation results with values that are ordered categorical responses (e.g. detected/not detected, present/absent) as opposed to "nominal" results where values do not provide quantitative information or rank ordering (e.g. names of bacteria). The second group is a quantitative measurement with a numeric result. This difference was modelled in two different FHIR profiles: One for organisms' detection, with the element 
value which contains the test result, being a coded concept (e.g. organism's name); the other profile was modelled to define quantitative observations with a numeric result. SNOMED CT provides codes for many test results where non-numeric answers are required, and it includes the most complete and reliable collection of concepts for names of microorganisms.

Most tests included in the dataset depend on the detection of the presence of the organism. For this reason, it seemed appropriate to link all observations to the detection. FHIR profiles are stand-alone packets of information but there are several possibilities of creating connections between them. We decided to use the hasMember element, which enables to group observations together while also creating a parental structure. The Observation for the detection of an organism was thus considered the parent observation targeting other observations in the hasMember element.

An important laboratory test also included in the dataset is the bacterial susceptibility test panel with both quantitative and qualitative results. These assays represent a rare type of LOINC terms that enable either ordinal results such as Sensitive, Intermediate, or Resistant $(S, I, R)$ or numeric values for the minimum inhibitory concentration. This test panel was identified with the code 50545-3 Bacterial susceptibility panel by Minimum inhibitory concentration (MIC). The panel consists of a group of observations where the sensitivity of bacteria to a number of antibiotics is measured. Each single measurement is modelled in a FHIR Observation where the result can be reported either as quantitative in the value element with an interpretation element for the ordinal result $(S, I, R)$ or have the ordinal result in value. [9]

The panel Observation itself does not expect a result of its own. It is rather a container for the specific antibiotic measurements. For this reason, we have used again the hasMember relationship to link the single antibiotic test observations to the panel observation. We have thus implemented a two-level observation structure as shown in figure 2 .

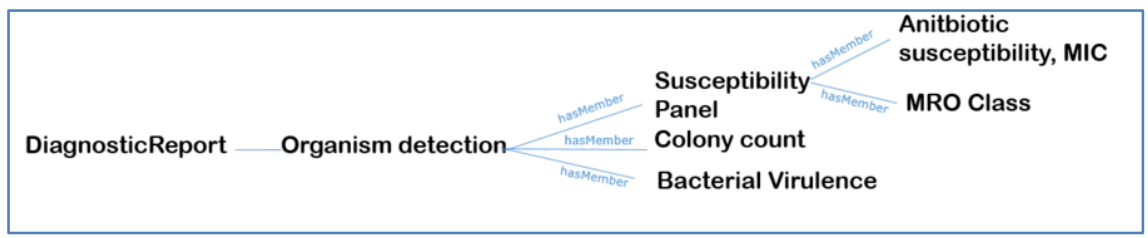

Figure 2. The Observation structure for the microbiology observation of the use case Infection Control

This proposal for a microbiology observation structure is specific to the dataset of the use case, but could be applied to other microbiology observations and could contain further nested information as shown in figure 3.

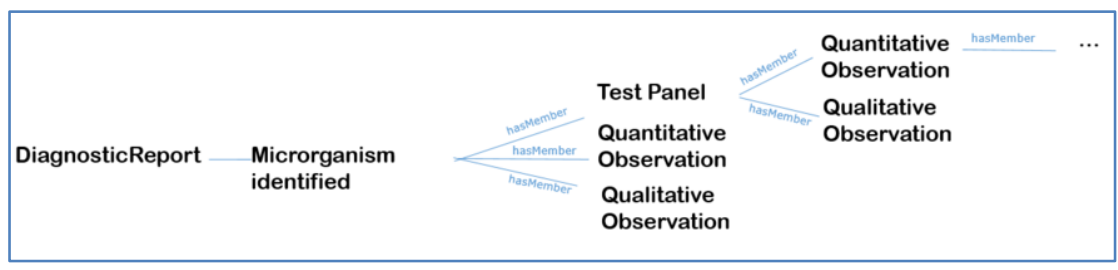

Figure 3. An example of a nested observation structure for microbiology observations 
In the end, seven observation profiles were modeled and uploaded onto Simplifier. [10]: Bacteria Detection, MRO Class, Colony Count, Susceptibility Panel, Antibiotic specific susceptibility, Resistance mechanism, and Bacterial Virulence. Within the HiGHmed Project, also an implementation guide is being developed in Simplifier in order to support the understanding and possible application of the profiles.

The ServiceRequest profile was used to model the information of the microbiology dataset such as the requester identifier and the laboratory inquiry. Moreover, the DiagnosticReport that reports the findings and interpretation of diagnostic tests, references the ServiceRequest through the element basedOn. This element is a mandatory field in the general MII DiagnosticReport profile.

\section{Lessons learned (Discussion)}

We have modeled the use case microbiology data using the standard FHIR and using international standard terminologies which are appropriate to describe laboratory tests and results.

In FHIR, semantic information is strictly connected to the data structure; therefore the profiles created were strongly based on the terminology system selected. To enable wider interoperability it is therefore essential to select the widely used international standards whenever possible.

However, this might also not be practicable such as in the case of a classification for multidrug-resistant organisms (MRO Class). This MRO Class is a local classification which includes terms published by the Commission for Hospital Hygiene and Infection Prevention (KRINKO) at the Robert Koch Institute (RKI), Germany,[11] and we did not find these terms in the international standard terminologies.

The elements of the MRO Class value set that were missing from the international standard terminologies were submitted to SNOMED CT as new concepts, and two were accepted.

While we succeeded in having two new terms included in the next SNOMED CT international edition, other terms belonging to the RKI Classification were considered not within the scope of the SNOMED description logic and not aligned with other national guidelines. Therefore, we could not use SNOMED CT terminology to define the MRO Class. Instead, we created a specific FHIR CodeSystem and ValueSet according to the RKI classification and associated it with the specific observation profile.

\section{Conclusion}

Results coming from medical laboratory examinations are one of the most fundamental tools of clinical diagnostics for therapy decisions and for the surveillance and prevention of infectious diseases.

For microbiology data, the use of LOINC and SNOMED CT for terminology serves the purpose of describing the tests, the results and the specimen information. The FHIR profiles implemented for the microbiology examinations constitute a model that supports syntactic and semantic interoperability. When standard terminologies do not include the required terms, it is possible to submit requests for new codes and thus contribute to the maintenance and completeness of the systems and further support interoperability. 


\section{Conflict of Interest}

The authors state that they have no conflict of interests.

\section{References}

[1] X. Gansel, M. Mary, and A. van Belkum, Semantic data interoperability, digital medicine, and e-health in infectious disease management: a review, Eur J Clin Microbiol Infect Dis. 38 (2019) 1023-1034. doi:10.1007/s10096-019-03501-6.

[2] B. Haarbrandt, B. Schreiweis, S. Rey, U. Sax, S. Scheithauer, O. Rienhoff, P. Knaup-Gregori, U. Bavendiek, C. Dieterich, B. Brors, I. Kraus, C.M. Thoms, D. Jäger, V. Ellenrieder, B. Bergh, R. Yahyapour, R. Eils, H. Consortium, and M. Marschollek, HiGHmed - An Open Platform Approach to Enhance Care and Research across Institutional Boundaries, Methods Inf Med. 57 (2018) e66-e81. doi:10.3414/ME18-02-0002.

[3] S. Semler, F. Wissing, and R. Heyder, German Medical Informatics Initiative: A National Approach to Integrating Health Data from Patient Care and Medical Research, Methods Inf Med. 57 (2018) e50-e56. doi:10.3414/ME18-03-0003.

[4] O. Bodenreider, R. Cornet, and D.J. Vreeman, Recent Developments in Clinical Terminologies SNOMED CT, LOINC, and RxNorm, Yearb Med Inform. 27 (2018) 129-139. doi:10.1055/s-00381667077.

[5] S. Sabutsch, and G. Weigl, Using HL7 CDA and LOINC for standardized laboratory results in the Austrian electronic health record, LaboratoriumsMedizin. 42 (2018) 259-266. doi:10.1515/labmed2018-0105.

[6] M.V. Andersen, I.H. Kristensen, M.M. Larsen, C.H. Pedersen, K.R. Gøeg, and L.B. Pape-Haugaard, Feasibility of Representing a Danish Microbiology Model Using FHIR, Stud Health Technol Inform. 235 (2017) 13-17.

[7] SIMPLIFIER.NET - Medizininformatik Initiative, (n.d.). https://simplifier.net/organization/ koordinationsstellemii (accessed July 17, 2020).

[8] B.E. Dixon, J. Hook, and D.J. Vreeman, Learning From the Crowd in Terminology Mapping: The LOINC Experience, Lab Med. 46 (2015) 168-174. doi:10.1309/LMWJ730SVKTUBAOJ.

[9] Guide for Using LOINC Microbiology Terms, LOINC. (n.d.). https://loinc.org/guides/micro/ (accessed July 16, 2020).

[10] SIMPLIFIER.NET - Medizininformatik Initiative - HiGHmed - IC, (n.d.). https://simplifier.net/ MedizininformatikInitiative-HiGHmed-IC (accessed July 17, 2020).

[11] M. Kaase, MRGN: New classification for multidrug-resistant Gram-negative bacteria, Journal of Laboratory Medicine. 37 (2016). doi:10.1515/labmed-2014-0004 